\title{
Therapeutic Strategies for Untreated Capillary Malformations of the Head and Neck Region: A Systematic Review and Meta-Analyses
}

\author{
Gonca Cinkara ${ }^{1} \cdot$ Ginger Beau Langbroek ${ }^{2}\left(\mathbb{D}\right.$. Chantal M. A. M. van der Horst ${ }^{3}$ - Albert Wolkerstorfer ${ }^{4}$. \\ Sophie E. R. Horbach ${ }^{3}$. Dirk T. Ubbink ${ }^{2}$
}

Accepted: 2 June 2021 / Published online: 23 June 2021

(c) The Author(s) 2021

\begin{abstract}
Background Capillary malformations of the head and neck region often cause psychological and physical burden. As the effectiveness of modern laser and light therapies is still suboptimal, patients often seek different therapeutic strategies. Other recognized, but not routinely proposed therapies include cosmetic camouflage, surgery, and medical tattooing. Information on therapeutic outcomes is currently lacking for patients to adequately participate in the treatment decision-making process. Objective The objective of this systematic review was to review the effectiveness and safety of recognized therapies for untreated capillary malformations of the head and neck: laser and light treatment modalities, photodynamic therapy, cosmetic camouflage, medical tattooing, and surgery.

Methods PubMed, Embase, and the Cochrane Central Register of Controlled Trials were searched up to 16 December, 2020 for observational and experimental studies examining recognized therapies for untreated capillary malformations of the head and neck. Two reviewers independently evaluated the risk of bias of included studies. Predefined treatment and safety outcomes of pooled data were scored using the Grading of Recommendations Assessment, Development and Evaluation (GRADE).

Results We included 48 observational and three randomized studies (totaling 3068 patients), evaluating nine different therapies. No studies on surgery or cosmetic camouflage matched our inclusion criteria. The pooled proportion of patients reaching a $\geq 75 \%$ clearance was $43 \%\left(95 \%\right.$ confidence interval $\left.24-64 \% ; I^{2}=55 \%\right)$ for the pulsed dye laser after three to eight treatment sessions (GRADE score: very low). Other therapies were less effective. Hyperpigmentation was most frequently described after the pulsed dye laser (incidences up to 40\%). Pain was most common after photodynamic therapy, yet the intensity was unreported. Substantial heterogeneity among studies as to patient characteristics and outcomes limited pooling and data comparisons.

Conclusions The pulsed dye laser seems preferable for treatment-naive capillary malformations of the head and neck region, yet demonstrates greater hyperpigmentation rates compared with other therapies. Our results are, however, based on lowquality evidence. Future studies using uniform outcome measures and validated metrics are warranted for study comparability. Based on this systematic review, clinicians and patients should be aware of the limited evidence about the available options when making (shared) treatment decisions for capillary malformations.
\end{abstract}

Trial Registration Review registration number PROSPERO database: CRD42020199445.

Gonca Cinkara and Ginger Beau Langbroek contributed equally to this review.

Ginger Beau Langbroek

g.b.langbroek@amsterdamumc.nl

Extended author information available on the last page of the article

\section{Introduction}

Capillary malformations (CMs, a.k.a port wine stains) are characterized by hyperdilated capillaries and post-capillary venules of the dermis [1]. These congenital lesions occur in $0.04-2.1 \%$ of newborns and have been associated with somatic mosaic and germline mutations in the GNAQ, GNA11, PIK3CA, RASA1, and EPHB4 genes [2-7]. They manifest as flat pink macules to more hypertrophic red-topurple lesions [8]. 


\section{Key Points}

Pulsed dye laser is recommended for treatment-naive capillary malformations of the head and neck region, but demonstrates greater hyperpigmentation rates compared with other therapies.

The effectiveness of surgery and medical camouflage for capillary malformations remains unclear.

Because of substantial heterogeneity in outcomes, we advocate for the development of uniform outcomes and validated outcome measurement instruments in prospective studies evaluating treatments for capillary malformations.

In addition to bleeding due to the development of vascular blebs, their disfiguring appearance may also greatly affect the patients' quality of life, particularly because CMs often occur in the head and neck [9-15]. An important reason for treating CMs is therefore to inhibit progression leading to hypertrophy and lessen functional complications.

Yet, despite technological evolution, the effectiveness of modern laser and light therapies, including the pulsed dye laser (PDL) as the current treatment of choice, has not improved over recent decades [16]. As CM lesions may recur post-therapy, patients are left with a desire for more effective treatment options. Other recognized therapies include cosmetic camouflage, surgical excision, and medical tattooing [17-19]. However, the latter treatment options are not routinely proposed to patients in the initial treatment decision-making process.

To adequately participate in this decision-making process, patients with CMs (and/or their parents) need to be well informed about the expected outcomes of all possible treatment options. Essential information seems to be lacking as, to date, there has not been a systematic comparison of the effectiveness of abovementioned therapies. More importantly, there is currently no international consensus on treatment guidelines for CMs.

The aim of this systematic review is to systemically compare the evidence of available therapeutic strategies for treatment-naive (untreated) CMs of the head and neck region to strengthen the treatment decision-making process.

\section{Materials and Methods}

This systematic review was conducted according to the guidelines of the Preferred Reporting Items for Systematic Reviews and Meta-Analyses (PRISMA) statement and the
Meta-analyses of Observational Studies in Epidemiology (MOOSE) guidelines [20, 21]. The research protocol was registered in the PROSPERO database (CRD42020199445).

\subsection{Literature Search}

PubMed (MEDLINE), EMBASE, and Cochrane Central Register of Controlled Trials (CENTRAL) databases were searched for published studies reporting on therapeutic effectiveness in patients with CMs from inception to 16 December, 2020. The search strategy was built with the support of a medical librarian and included the following MeSH (Medical Subject Heading) terms: 'Port-Wine Stain,' 'Lasers,' 'Laser therapy,' 'Surgical procedures, operative,' 'Photochemotherapy,' and 'Tattooing'. Relevant keywords and synonyms describing the condition and different types of therapies, including camouflage, were augmented (see Table 1 of the Electronic Supplementary Material [ESM] for the search strategy). Reference lists of all included articles were screened for additional relevant articles. We did not contact authors for additional data.

\subsection{Study Selection}

The retrieved articles were entered and deduplicated in EndNote X9. Study selection was performed independently by two researchers (GC and $\mathrm{GL})$. A third reviewer $(\mathrm{CH})$ was consulted to resolve any disagreement.

\subsubsection{Inclusion Criteria}

Original studies with all study designs reporting on therapeutic effectiveness of laser or light therapy, photodynamic therapy (PDT), camouflage, surgery, or medical tattooing of previously untreated CMs in the head or neck region were included. We included therapeutic studies published since the year 2000 only for laser or light therapy and PDT because around that time alternative light sources became available with the introduction of the neodymium-doped yttrium aluminum garnet/potassium titanyl phosphate (Nd:YAG/KTP) laser and intense pulsed light (IPL) [16]. Studies with no documentation of previous therapies were included. Furthermore, no publication language restriction was applied. Publications other than English were translated with Google Translate.

\subsubsection{Exclusion Criteria}

Case reports with fewer than five patients were excluded from this review to strengthen the generalizability of results. Studies were excluded if the article did not report on our prespecified outcome measures or if combinations of therapies 
were applied. All inclusion and exclusion criteria are shown in Table 1.

\subsection{Pre-Specified Outcome Measures}

The following outcome measures of interest were predefined: effectiveness as the primary outcome, described as any quantitative improvement of the CM, e.g., Physician Global Assessment or Patient Global Assessment, clearance or improvement reported as percentage ranges, change in $L^{*} a^{*} b^{*}$ color coordinate system values [22], or changes in the erythema index. Pre-specified secondary outcomes included patient satisfaction and complications related to the procedure: hyperpigmentation, hypopigmentation, scarring, and pain. Other reported complications are also described, but not considered for meta-analyses.

\subsection{Data Extraction}

The two reviewers (GC and GL) independently extracted the following study characteristics and patient data from each included study, using a predefined digital data extraction form: authors, publication year, study design, number of patients, mean age, Fitzpatrick skin type, characteristics of the CM (color, size, hypertrophy), pretreatment, type of treatment and corresponding characteristics, number of treatment sessions, treatment interval, data on predefined outcome measures as previously described, and follow-up duration.

\subsection{Risk of Bias Assessment}

Two reviewers independently assessed the risk of bias (GC and GL). For non-randomized studies, the Dictionary of the Effective Public Health Practice Project tool was used
[23]. Consistent with the Dictionary Component Rating Scale, the tool domains were rated as 'strong,' 'moderate,' and 'weak'. Randomized clinical trials were evaluated by means of the critical appraisal checklist issued by the Dutch Cochrane Collaboration [24]. The Grading of Recommendations Assessment, Development and Evaluation (GRADE) approach was used to rate the quality of the evidence for the pre-specified outcomes that could be pooled [25].

\subsection{Data Analysis}

Extracted data were presented whenever possible as means with standard deviations, medians with interquartile ranges, or percentages with $95 \%$ confidence intervals. Meta-analyses of proportions or means using a random-effects model were performed if studies showed similar patient and treatment characteristics. If statistical heterogeneity was high $\left(I^{2}>\right.$ $75 \%$ ) or fewer than five studies were available for a specific outcome, no meta-analyses would be conducted, but study results would be explored and summarized as forest plots without totals.

To enable meta-analyses, the different outcome measures used for improvement of the CM or investigator/physician/ patient global assessment scores were converted into dichotomous scales: 'poor/moderate/good' $\leq 75 \%$ improvement and 'excellent' $=\geq 75 \%$ improvement. This could only be done if studies described their results as quartiles of percentage lightening (i.e., $0-25 \%, 25-50 \%, 50-75 \%$, and $75-100 \%)$.

Studies that used other percentage ranges that could be converted to the aforementioned categorical scales were also included in the meta-analyses. Differences are presented as risk differences with their 95\% confidence intervals. Studies that quantitatively reported qualitative effects were converted into the 'poor/moderate/good' $\leq 75 \%$ improvement

Table 1 Study selection criteria

\begin{tabular}{|c|c|c|}
\hline & Inclusion criteria & Exclusion criteria \\
\hline $\begin{array}{l}\text { All therapies (laser or light treatment } \\
\text { modalities, photodynamic therapy, } \\
\text { camouflage, surgery, or medical } \\
\text { tattooing) }\end{array}$ & $\begin{array}{l}\text { Patients of all ages with CMs in the head or neck region, } \\
\text { including disorders associated with CMs } \\
\text { CMs of any color and size, including hypertrophic and } \\
\text { non-hypertrophic lesions } \\
\text { All study designs } \\
\text { Articles describing the quantitative improvement of CMs } \\
\text { Studies including } \geq 5 \text { patients }\end{array}$ & $\begin{array}{l}\text { CMs in other body parts } \\
\text { Acquired/traumatic CMs } \\
\text { No full text available } \\
\text { Conference abstracts } \\
\text { Unpublished literature } \\
\text { In vivo studies } \\
\text { Animal studies } \\
\text { Combination of therapies for the same CM } \\
\text { Pre-treated CMs }\end{array}$ \\
\hline $\begin{array}{l}\text { Laser or light treatment modalities, } \\
\text { photodynamic therapy }\end{array}$ & Publication year $\geq 2000$ & \\
\hline $\begin{array}{l}\text { Camouflage, surgery, or medical } \\
\text { tattooing }\end{array}$ & No restriction on publication date & \\
\hline
\end{tabular}


group when the following terms were used: poor, fair, moderate, partial, slight improvement, a bit better, quite a bit better, satisfactory, good, and a lot better. Likewise, results with terms like very good, very satisfying, excellent, or clear were categorized in the 'excellent' $(\geq 75 \%)$ group.

All other pre-specified outcomes were dichotomized as 'yes' or 'no'. Data from studies that could not be pooled were displayed separately and explored in a descriptive manner. Meta-analyses were performed using Rstudio Version 1.3.1056 (Rstudio PBC, Boston, MA, USA).

\section{Results}

\subsection{Included Studies}

The search generated a total of 4200 hits. After eliminating duplicates, 2894 studies were screened based on titles and abstracts. Next, 318 articles were screened in full text and 51 of these studies met the inclusion criteria [26-76]. Details about study selection are described in Fig. 1 of the ESM.

\subsection{Study Characteristics}

Of the 51 included studies, 50 studies adequately described the number of included patients with $\mathrm{CMs}$ of the head and neck region and comprised a total of 3068 patients. From one study, only the number of treated CM lesions of the head and neck region could be derived [27]. Out of the 51 studies, 48 were prospective or retrospective cohort studies and three were randomized clinical trials. The study characteristics per treatment modality are shown in Table 2 of the ESM. The number of participants per study ranged from 5 to 306 patients, with mean ages from 3 months to 39.5 years. Most patients had Fitzpatrick skin types I-IV and CM lesion colors varied from pale pink to dark red and purple. The following treatments were encountered: PDL, Nd:YAG laser, KTP, KTP+; $\mathrm{CO}_{2}$ laser; 577-nm (yellow) laser; dual sequential wavelength laser, IPL, PDT, and medical tattooing. Photodynamic therapy was studied alone or compared to other laser modalities. No studies on surgery or cosmetic camouflage were included, as they did not meet the eligibility criteria (i.e., largely pretreated patients, effectiveness not quantitatively described, mix of vascular malformations) or lacked adequate outcome reporting. Treatment characteristics varied greatly between and within studies. For example, for PDT, two different types of photosensitizers were used: hematoporphyrin monomethyl ether or PsD-007. Furthermore, the total number of treatments (range 1-15), treatment interval (a few weeks to several months), and follow-up (range 4 weeks -5 years) varied substantially.

\subsection{Risk of Bias Assessment}

The overall methodological quality of the included nonrandomized studies (48 out of 51 studies) was poor, mainly owing to the study design, no correction for confounding variables (e.g., age, sex, skin type, CM characteristics), and use of non-validated and non-reliable outcome measurement instruments for CMs. The three randomized clinical trials contained blinding of outcome assessments, but displayed potential risks of selection bias and two randomized clinical trials lacked information on blinding of patients and physicians. The risk of bias assessment of the included studies is summarized in Fig. 2 of the ESM.

\subsection{Treatment Outcomes}

Table 2 of the ESM shows the outcome measures the studies intended to report. The results of the predefined outcomes of the individual studies are summarized per treatment modality in Figs. 1, 2 and 3 and in Table 3 of the ESM. Only a limited number of studies reported on our predefined outcomes and because of the large heterogeneity in outcomes, pooling of most outcomes was impossible.

\subsubsection{PDL}

Most studies addressed PDL, with 1697 patients involved in 28 studies, and $674 \mathrm{CM}$ lesions in one study. The pooled estimate of the proportion of patients reaching a $\geq 75 \%$ clearance was $43 \%$ (95\% confidence interval $24-64 ; I^{2}=55 \%$ ) after three to eight treatment sessions (Fig. 1a). Overall, more 'excellent' clearance scores ( $\geq 75 \%$ clearance) were achieved in infants, with a range of $63-90 \%$ in three studies (89 patients in total) after a mean of three to nine treatment sessions [31, 34, 39]. A significant correlation was found between objective and subjective treatment outcomes in the study by Fallahi et al., in which the mean Investigator Global Assessment score was $64.33 \pm 23.67 \%$ and the mean Patient Global Assessment score was $65 \pm 20.44 \%$ (correlation coefficient: $0.85, p<0.001$ ) after four treatment sessions [38]. Treatment outcomes of the remaining studies examining PDL were reported as mean clearance or color improvement scores, $L^{*} a^{*} b^{*}$ color coordinate system values, or Visual Analogue Scale (VAS) scores. Hyperpigmentation was the most frequent adverse event, with incidences between 0 and $41 \%$ (Fig. 2a). One study, involving ten patients with Fitzpatrick skin type V, reported a $20 \%$ incidence of scarring after PDL therapy with a follow-up duration of 49 months (Fig. 3a) [63]. In general, patients were satisfied with treatment outcomes $[43,55]$. 


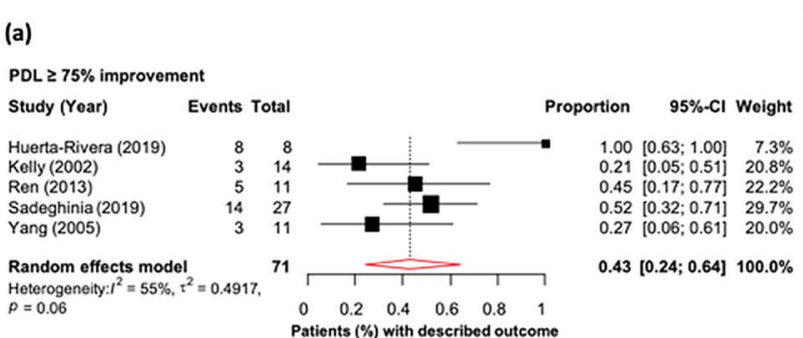

(b)

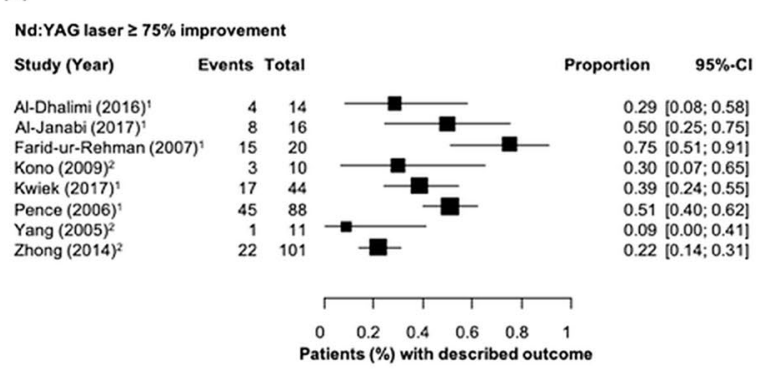

(c)

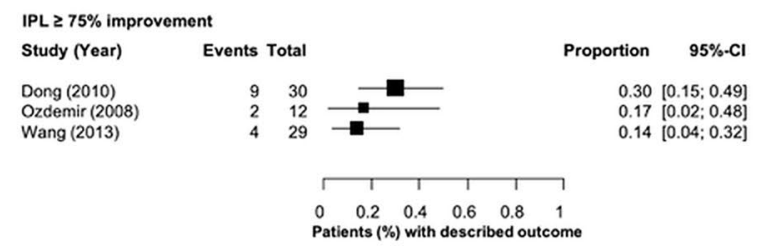

Fig. 1 a-f Effectiveness as the proportion of patients who reached $\geq 75 \%$ clearance. a Pooled estimated effectiveness for the pulsed dye laser (PDL) of studies with three to eight treatment sessions, Fitzpatrick skin types I-IV, and age $>1$ year. b-f Unpooled (range of) effectiveness of neodymium-doped yttrium aluminum garnet (Nd:YAG)

\subsubsection{Nd:YAG Laser}

Eight studies evaluated Nd:YAG laser treatment in 304 patients. With a 532-nm Nd:YAG laser, $9-75 \%$ of the patients reach a $\geq 75 \%$ improvement after 3-12 treatment sessions. The 1064-nm Nd:YAG laser was less effective with $9-30 \%$ of the patients reaching a $\geq 75 \%$ improvement (Fig. 1b) and patients were less satisfied compared with the 532-nm laser (satisfaction scores 7.6 [standard deviation $=$ 2.3 ] vs 3.3 [standard deviation $=0.8$ ],$p<0.001$, respectively) [29]. Overall, low adverse event rates were reported (Figs. $2 \mathrm{~b}$ and $3 \mathrm{~b}$ ). One study reported a hyperpigmentation rate of $65 \%$, but this was only transient [37]. Scarring occurred in $0-9 \%$ of the patients.

\subsubsection{IPL}

The IPL laser was examined in only three studies, comprising 71 patients. Between $14 \%$ and $30 \%$ of adult patients reached 'excellent' clearance after a mean of five treatment sessions (Fig. 1c). Adverse events were reported in the (d)

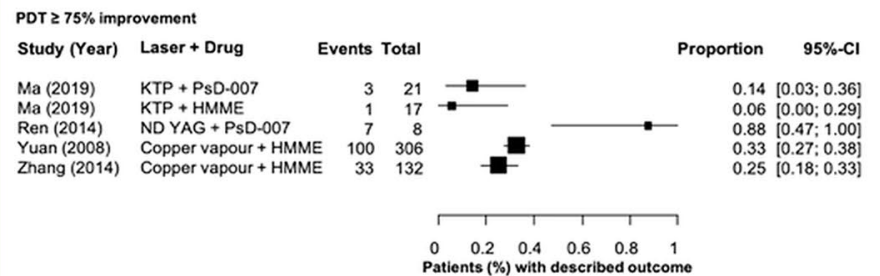

(e)

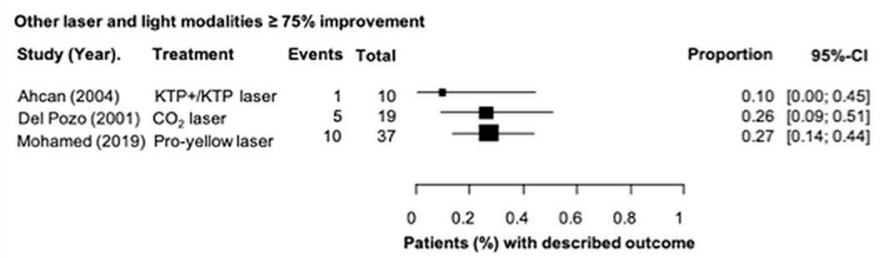

(f)

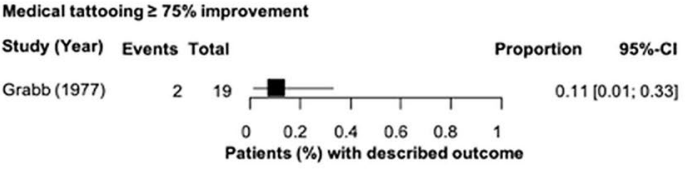

laser, intense pulsed light (IPL), photodynamic therapy, other laser and light modalities, and medical tattooing. $\tau^{2}$ dispersion, $C I$ confidence interval, $I^{2}$ heterogeneity, $p$ probability value, ${ }^{1} 532$ nanometer, ${ }^{2} 1064$ nanometer

study by Özdemir et al., who observed permanent hypopigmentation $(n=1)$ and hypertrophic scarring $(n=2)$ in adult patients with a follow-up between 6 and 13 months (Figs. 2c and 3c) [54]. Wang et al. reported pain in $100 \%$ of the patients [66] (Fig. 3c).

\subsubsection{PDT}

Seven studies, comprising 865 patients, examined PDT. In two prospective cohort studies on PDT with a lightemitting diode source and hematoporphyrin monomethyl ether, between 6 and $31 \%$ of the patients achieved almost complete clearance ( $>90 \%$ ) after one to two treatment sessions [51,68]. Overall, between 6 and $88 \%$ of the patients reached a $\geq 75 \%$ improvement after one to four treatment sessions with different photosensitizers and light sources (Fig. 1d). Adverse events were infrequently reported. Yet, pain occurred in $100 \%$ of the patients in two studies, but pain intensity was not reported (Fig. 3d) [51, 52]. Furthermore, scarring was present in 3-4\% of the patients in two studies $[72,75]$. 
(a) PDL Hyperpigmentation

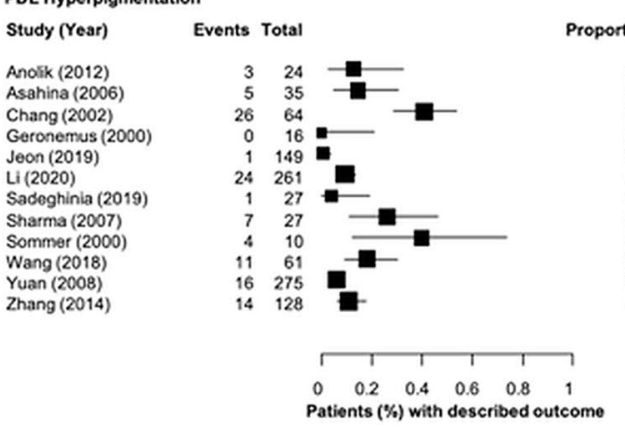
Patients $(\%)$ with described outcom (b) Nd:YAG laser Hyperpigmentation Study (Year) Events Total Al-Dhalimi (2016)' Farid-ur-Rohman (2007) Kono $(2009)^{2}$ Pence (2006)
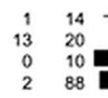

$\sqrt{1}$

POL Hypopigmentation
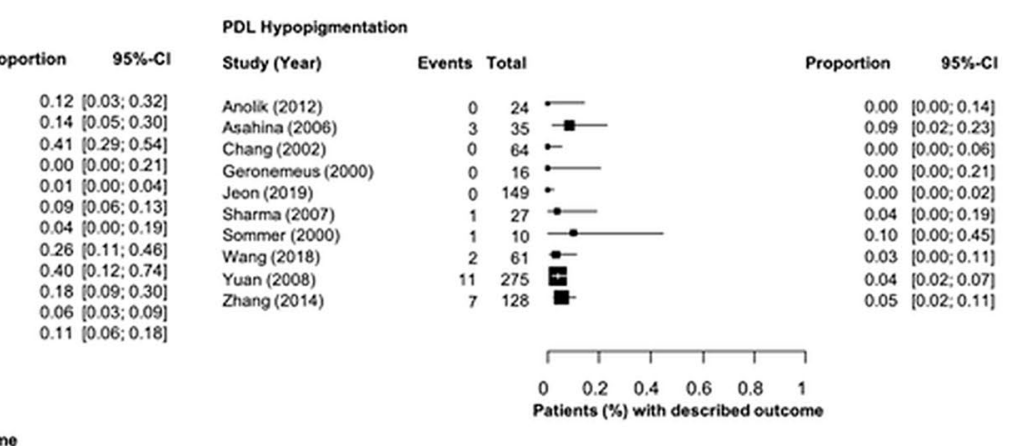

C.CI
$0.32]$
$0.30]$
0.54 ]
$0.21]$
$0.04]$
$.13]$
$0.19]$
$0.46]$
$0.74]$
$0.09]$
$0.18]$

Nd:YAG laser Hypopigmentation

Study (Year) Events Total

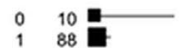

Proportion 95\%.Cl $\begin{array}{ll}0.07[0.00 ; 0.34] & \text { Kono }(2009)^{2} \\ 0.65[0.41 ; 0.85] & \text { Pence }(2006)^{\prime}\end{array}$ $0.00[0.00 ; 0.31]$

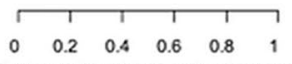

Patients (\%) with described outcome

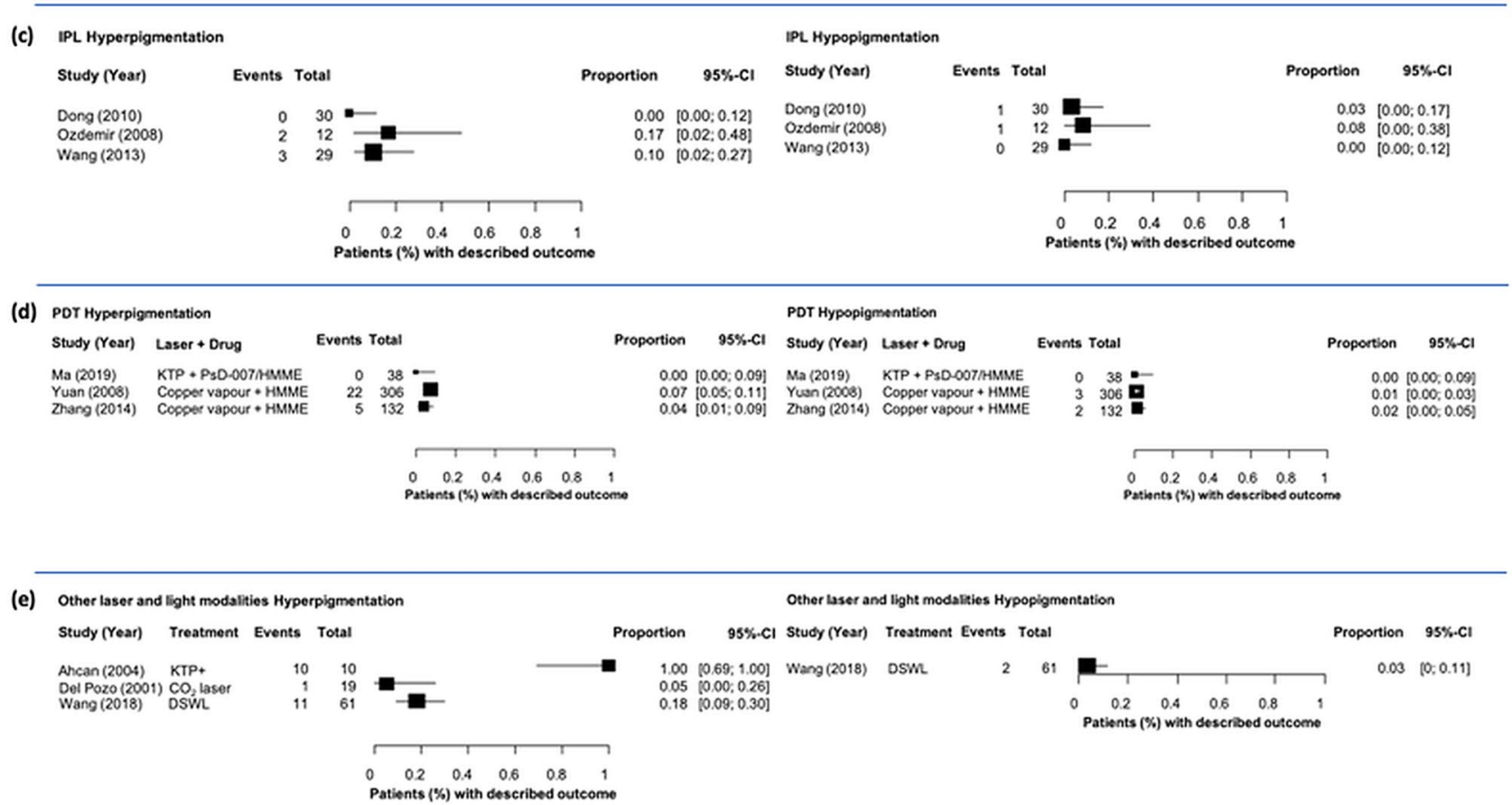

Fig. 2 a-e Predefined adverse events of hyperpigmentation and hypopigmentation per treatment modality. a Pulsed dye laser (PDL); b neodymium-doped yttrium aluminum garnet (Nd:YAG laser); c intense pulsed light (IPL); d photodynamic therapy (PDT); and e other laser and light modalities. No adverse events were reported for

\subsubsection{Other Laser and Light Modalities}

Five studies (138 patients) were included using other laser and light modalities: the KTP+/KTP laser, the $\mathrm{CO}_{2}$ laser, a 577-nm (pro-yellow) laser, and a dual sequential wavelength laser. The $\mathrm{CO}_{2}$ laser was applied for hypertrophic $\mathrm{CMs}$ with medical tattooing. $\mathrm{CI}$ confidence interval, $\mathrm{CO}_{2}$ carbon dioxide, $\mathrm{DSWL}$ dual sequential wave length, $H M M E$ hematoporphyrin monomethyl ether, KTP potassium titanyl phosphate, $P s D-007$ second-generation photosensitizer, ${ }^{1} 532$ nanometer, ${ }^{2} 1064$ nanometer

nodules. These lasers revealed inferior results to the PDL, as only $10-27 \%$ of the patients achieved 'excellent' $(\geq 75 \%)$ clearance scores (Fig. 1e) [28, 35, 53]. Hyperpigmentation and scarring were most common after the KTP+ and KTP laser (100\% vs 20\%, respectively) (Figs. 2e and 3e). 
(a)

PDL Scarring
Study (Year)
Anolik (2012)
Chang (2002)
Chapas (2007)
Geronemus (2000)
Jeon (2019)
Kelly (2002)
Li (2020)
Sadeghinia (2019)
Sharma (2007)
Sommer (2000)
Wang (2018)
Yuan (2008)
Zhang (2014)

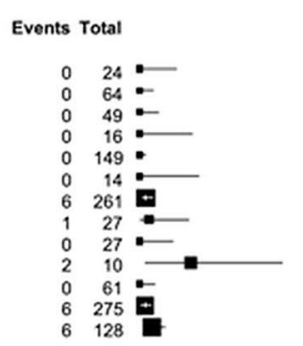

Proportion $\quad 95 \%-\mathrm{Cl}$

$0.00[0.00 ; 0.14]$ $0.00[0.00 ; 0.06]$ $0.00[0.00 ; 0.07]$ $0.00[0.00 ; 0.02]$ $0.00[0.00 ; 0.23$ $0.02[0.01 ; 0.05]$ $0.04[0.00: 0.19]$ $0.00[0.00 ; 0.13$ $0.20[0.03 ; 0.56]$ $0.00[0.00 ; 0.06$ $0.02[0.01 ; 0.05$ $0.05[0.02 ; 0.10]$

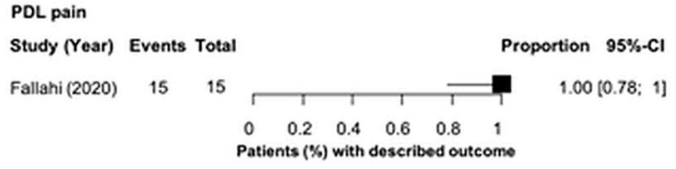

(b) Nd:YAG laser Scarring
Study (Year)
Events Total
Proportion $\quad 95 \%$ CI
A-Dhalimi (2016)"2 Kono $(2009)^{2}$ Kwiek (2017) Yang $(2005)^{2}$

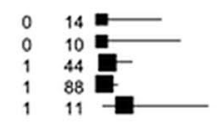
$0.00[0.00 ; 0.23]$
$0.00[0.00 ; 0.31]$
$0.0110 .00 ; 0.06$
$0.09[0.00 ; 0.41]$

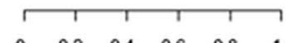
$\begin{array}{llllll}0 & 0.2 & 0.4 & 0.6 & 0.8 & 1\end{array}$
Patients (\%) with described outcome

(c)

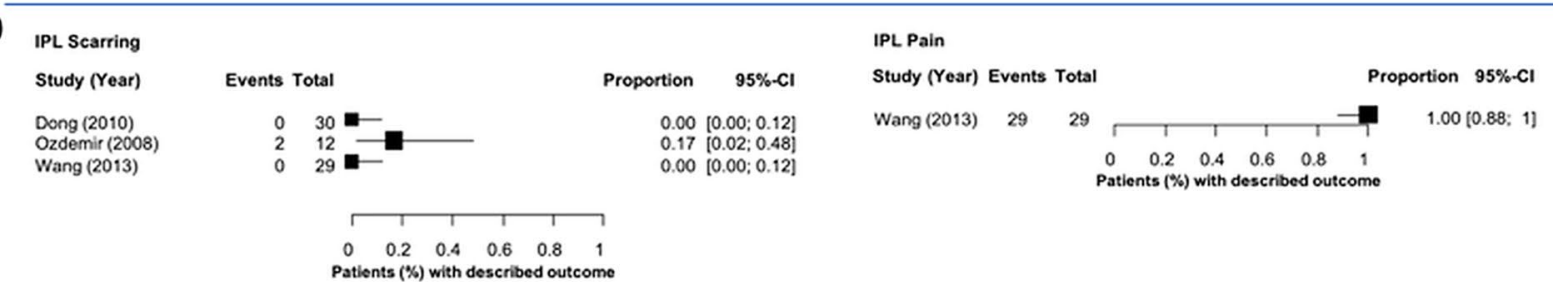

(d) Por Scarring

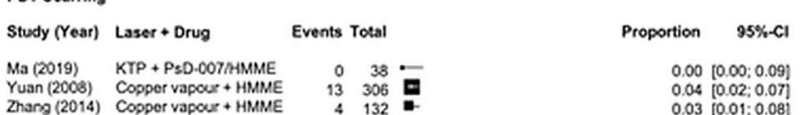

PDT Pain

Study (Year) Li-Qiang (2018)
Ma (2019)

Events Total

Proportion 95\%-Cl

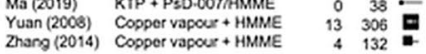
$0.03[0.01 ; 0.08]$ $\begin{array}{ll}77 & 77 \\ 38 & 38\end{array}$ $1.00[0.95 ; 1]$

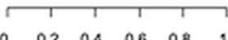
$\begin{array}{cccccc}0 & 0.2 & 0.4 & 0.6 & 0.8 & 1 \\ \text { Patients }(\%) & \text { with described outcome }\end{array}$

(e) Other laser and light modalitios Scarring

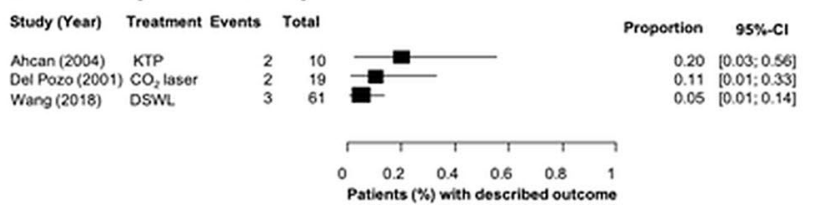

Fig. 3 a-e Predefined adverse events of scarring and pain per treatment modality. a Pulsed dye laser (PDL); b neodymium-doped yttrium aluminum garnet (Nd:YAG) laser; $\mathbf{c}$ intense pulsed light (IPL); d photodynamic therapy (PDT); and e other laser and light modalities. No adverse events were reported for medical tattooing. $C I$ confidence interval, $\mathrm{CO}_{2}$ carbon dioxide, $D S W L$ dual sequential wave length, HMME hematoporphyrin monomethyl ether, KTP potassium titanyl phosphate, $P s D-007$ second-generation photosensitizer, ${ }^{1} 532$ nanometer, ${ }^{2} 1064$ nanometer

\subsubsection{Medical Tattooing}

Medical tattooing was investigated in only three studies, including 65 patients. Between three and ten treatment

sessions were needed and follow-up duration was up to 3.1 years. According to a patient/parent assessment panel in the study by Grabb et al., a $\geq 75 \%$ improvement was attained in two out of 19 patients (11\%) (Fig. 1f) [40]. Van der Velden 
et al. reported that all patients achieved good results without loss of pigment color with a mean follow-up of 19.8 months [65]. None of the individual studies reported on our predefined secondary outcomes.

\subsection{Overall Quality of the Evidence (GRADE)}

The overall quality of the evidence (GRADE) could only be determined for the PDL regarding effectiveness and was rated as very low (Table 4 of the ESM). This rating was mainly due to a high risk of bias in the outcome assessment and publication bias, use of non-validated outcome measurement instruments, relatively small sample sizes (imprecision), and the heterogeneity of treatment outcomes (inconsistency).

\section{Discussion}

This systematic review describes 51 studies, in which 3068 patients with treatment-naive CMs were treated with either laser or light therapy, PDT, or medical tattooing. The quantitative improvement of CM lesions and reported adverse events varied widely between studies and treatment modalities. Pulsed dye laser was most frequently used, but less than half of the patients receiving PDL showed $a \geq 75 \%$ improvement. Other therapies revealed to be less effective. Pulsed dye laser studies reported higher hyperpigmentation rates in the majority of the studies as compared with other treatment modalities. Overall, hypopigmentation and scarring were uncommon, but results are based on only a few studies. If reported, pain occurred frequently after PDT and IPL in both children and adults, but pain intensity was not adequately described. Patient satisfaction was rarely assessed.

With this systematic review, we aimed to provide a comprehensive overview of treatment effectiveness and the safety of frequently used therapies for untreated CMs of the head and neck region. This evidence is essential to support patients with $\mathrm{CMs}$ in the treatment decision-making process. However, as no studies on cosmetic camouflage and surgery could be included in this review, there is still insufficient evidence regarding all available therapies to adequately support patients with CMs in the treatment decision-making process. Particularly for cosmetic camouflage, it seems striking that little evidence on its effectiveness exists, as it is still commonly used by patients with CMs. Currently, surgical therapy is predominantly used for hypertrophic lesions, in which laser treatment has a limited beneficial effect [77, 78]. Novel, mostly experimental therapies do exist, such as laser therapy with a topical adjunct or site-specific pharmacolaser therapy $[79,80]$. These, however, constitute combinations of therapies, which were excluded from this review as their separate effects cannot be ascertained.
Previously published (systematic) reviews on CMs mainly compared different laser or light modalities and assessed therapeutic effectiveness [16, 81]. Van Raath et al. recently concluded that laser treatment outcomes have not improved over the last decades and that $30.5 \%$ of previously untreated patients achieve $75-100 \%$ clearance [16]. Our findings in treatment-naive patients with CMs demonstrate better outcomes for the PDL. This is conceivably owing to the fact that only studies with similar patient and treatment characteristics were included in our pooled analysis, resulting in more case-matched outcomes.

The diverging treatment characteristics between and within studies may account for the observed differences in treatment outcomes. Because of this heterogeneity in treatment characteristics, we were not able to associate treatment outcomes with the specific treatment settings (pulse duration, energy fluence, spot size, number of passes, treatment sessions and intervals). Furthermore, the differing treatment outcomes may also depend on patient characteristics (age, skin type) and CM lesion characteristics. There is some evidence that infants under the age of 1 year achieve better clearance scores, as more patients reach a $\geq 75 \%$ improvement $[31,34,39]$. Yet, contradictory results have been published [82-84]. Furthermore, Wimmershoff et al. demonstrated that only $2 \%$ of the patients of various ages reach a $>75 \%$ improvement after PDL treatment, which supposedly could be because of more difficult-to-treat darker skin types or fewer treatment sessions $[69,85]$. The authors, however, did not report on this aspect. Notably, the results of two observational studies indicate that dark skin types may cause a higher risk of hyperpigmentation and scarring after laser therapy [37, 63]. This may be explained by the fact that darkskinned patients are more prone to develop epidermal damage. As with higher pigmentation levels, the laser light is predominantly absorbed by melanin before reaching hemoglobin, which may subsequently lead to inflammatory effects and post-inflammatory dyspigmentation [86-88]. The Pakistani study, however, did not sufficiently document skin type [37]. Furthermore, not all studies reported if epidermal cooling was applied, which could explain some of the reported hyperpigmentation cases among studies $[35,37,44,63,72]$. Because of limited reporting of CM lesion characteristics, drawing conclusions about which treatment modality is best for hypertrophic CMs in particular is not safe.

In this systematic review and meta-analyses, the methodological quality of the included studies in general was low. This was mainly because confounding factors were rarely corrected for and there was a lack of validated outcome measurement instruments for CMs. More importantly, heterogeneity was high in terms of patient, CM lesion, and treatment characteristics among and within studies. Hence, only a few low-quality studies could be pooled. Both this heterogeneity and the resulting small subgroup sizes 
obstructed adequate subgroup analyses. Nevertheless, this review presents the best evidence currently available. As both observational and experimental studies were included, we have been able to collect evidence on therapeutic efficacy and safety.

Second, the authors dichotomized categorized treatment outcomes whenever possible. In many studies, however, different outcome measures were used. This prohibited pooling of the results and made a proper comparison between treatment modalities impossible. In addition, some qualitative effects were converted into quantitative outcomes to support the comparison of study results. Conversion of these outcomes is inevitably arbitrary and interpretation should be done with caution.

One of the strengths of this review is that we provide a comprehensive overview of the available treatment options for CMs, while previous reviews mostly focused on PDL only. Additionally, our study discloses the deficiencies in existing knowledge regarding CM therapeutic efficacy studies. As outcome reporting in clinical research on CMs is anything but uniform, the evidence of clinical studies on $\mathrm{CM}$ treatment is hard to compare. Moreover, we still know little about which outcomes are considered most relevant by patients with CMs. As a result, the impact of CM treatments cannot be assessed sufficiently and optimal strategies for managing CMs care are still to be desired, while urgently needed for this patient group [89].

It is of great importance that uniform and relevant characteristics regarding patients, CM lesions, and treatments are provided in studies, and results should be described per patient when characteristics vary widely. We are therefore currently preparing an e-Delphi study as part of the international COSCAM (Core Outcome Set for CApillary Malformations) project, to reach a consensus on which outcome domains should be measured and reported in clinical studies regarding CMs. This project, registered at the Core Outcome Measures in Effectiveness Trials (COMET) website (registration number 1599), is guided by the Cochrane SkinCore Outcome Set Initiative (CS-COUSIN) that supports the standardization of outcome measurement in dermatological clinical trials [90].

\section{Conclusions}

Based on the currently available evidence and in the context of lacking guidelines, PDL therapy is recommended for treatment-naive CMs of the head and neck region, but at the cost of greater hyperpigmentation rates compared with other therapies. Our results are, however, based on low-quality evidence. Larger high-quality comparative studies using reliable validated methods and uniform outcome measures are therefore warranted. Based on this systematic review, clinicians and patients should be aware of the limited evidence when deciding on the available treatment options for CMs.

Supplementary Information The online version contains supplementary material available at https://doi.org/10.1007/s40257-021-00616-5.

Acknowledgments We thank information specialist Mrs. F.S. van Etten-Jamaludin for assisting in the literature search procedures and Dr. Susan van Dieren for support regarding the meta-analyses.

\section{Declarations}

Funding No funding was received for the preparation of this article.

Conflicts of interest/Competing interests Gonca Cinkara, Ginger Beau Langbroek, Chantal M.A.M. van der Horst, Albert Wolkerstorfer, Sophie E.R. Horbach, and Dirk T. Ubbink have no conflicts of interest that are directly relevant to the content of this article.

Ethics approval Not applicable.

Consent to participate Not applicable.

Consent for publication Not applicable.

Availability of data and material All data generated or analyzed during this study are included in this published article and its supplementary information files.

Code availability Not applicable.

Authors' contributions GC and GBL contributed equally to this review, and were both involved in the conception of the study, data collection and analysis, drafting of the manuscript, and critical revision of the manuscript. CvdH was involved in the conception of the study, data analysis, and critical revision of the manuscript. AW and SERH contributed to the conception of the study and critical revision of the manuscript. DTU was involved in the conception of the study, data analysis, and critical revision of the manuscript. All authors give approval of the final manuscript to be published.

Open Access This article is licensed under a Creative Commons Attribution-NonCommercial 4.0 International License, which permits any non-commercial use, sharing, adaptation, distribution and reproduction in any medium or format, as long as you give appropriate credit to the original author(s) and the source, provide a link to the Creative Commons licence, and indicate if changes were made. The images or other third party material in this article are included in the article's Creative Commons licence, unless indicated otherwise in a credit line to the material. If material is not included in the article's Creative Commons licence and your intended use is not permitted by statutory regulation or exceeds the permitted use, you will need to obtain permission directly from the copyright holder. To view a copy of this licence, visit http://creativecommons.org/licenses/by-nc/4.0/.

\section{References}

1. Burrows P, Fishman S, Mulliken J, Young A. Mulliken et Young's vascular anomalies. New York: Oxford University Press; 2013. 
2. Shirley MD, Tang H, Gallione CJ, et al. Sturge-Weber syndrome and port-wine stains caused by somatic mutation in GNAQ. N Eng J Med. 2013;368:1971-9.

3. Kahana M, Feldman M, Abudi Z, Yurman S. Frequency of birthmarks in newborn. Int J Dermatol. 1995;34:704-6.

4. Eerola I, Boon LM, Mulliken JB, et al. Capillary malformation-arteriovenous malformation, a new clinical and genetic disorder caused by RASA1 mutations. Am J Hum Genet. 2003;73(6):1240-9.

5. Amyere M, Revencu N, Helaers R, et al. Germline loss-of-function mutations in EPHB4 cause a second form of capillary malformation-arteriovenous malformation (CM-AVM2) deregulating RAS-MAPK signaling. Circulation. 2017;136(11):1037-48.

6. Couto JA, Ayturk UM, Konczyk DJ, et al. A somatic GNA11 mutation is associated with extremity capillary malformation and overgrowth. Angiogenesis. 2017;20(3):303-6.

7. Vahidnezhad H, Youssefian L, Uitto J. Klippel-Trenaunay syndrome belongs to the PIK3CA-related overgrowth spectrum (PROS). Exp Dermatol. 2016;25(1):17-9.

8. Schneider BV, Mitsuhashi Y, Schnyder UW. Ultrastructural observations in port wine stains. Arch Dermatol Res. 1988;280:338-45.

9. Geronemus RG, Ashinoff R. The medical necessity of evaluation and treatment of port-wine stains. J Dermatol Surg Oncol. 1991;17(1):76-9.

10. Enjolras O, Mulliken JB. The current management of vascular birthmarks. Pediatr Dermatol. 1993;10:311-33.

11. Geronemus RG, Ashinoff R. The medical necessity of evaluation and treatment of port-wine stains. J Dermatol Surg Oncol. 1991;17:76-89.

12. van Drooge AM, Beek JF, van der Veen JW, et al. Hypertrophy in port-wine stains: prevalence and patient characteristics in a large patient cohort. J Am Acad Dermatol. 2012;67:1214-9.

13. Hagen SL, Grey KR, Korta DZ, Kelly KM. Quality of life in adults with facial port-wine stains. J Am Acad Dermatol. 2017;76:695-702.

14. Lanigan S, Cotterill J. Psychological disabilities amongst patients with port wine stains. Br J Dermatol. 1989;121:209-15.

15. Troilius A, Wrangsjö B, Ljunggren B. Potential psychological benefits from early treatment of port-wine stains in children. $\mathrm{Br} \mathbf{J}$ Dermatol. 1998;139:59-65.

16. van Raath MI, Chohan S, Wolkerstorfer A, et al. Port wine stain treatment outcomes have not improved over the past three decades. J Eur Acad Dermatol Venereol. 2019;33:1369-77.

17. Conway H. Tattooing of nevus flammeus for permanent camouflage. JAMA. 1953;152:666-9.

18. McMichael L. Skin camouflage. BMJ. 2012;344:d7921.

19. Clodius L. Surgery for the extensive facial port-wine stain? Aesthetic Plast Surg. 1985;9:61-8.

20. Stroup DF, Berlin JA, Morton SC, et al. Meta-analysis of observational studies in epidemiology: a proposal for reporting. Metaanalysis Of Observational Studies in Epidemiology (MOOSE) Group. JAMA. 2000;283:2008-12.

21. Moher D, Shamseer L, Clarke M, et al. Preferred reporting items for systematic review and meta-analysis protocols (PRISMA-P) 2015 statement. Syst Rev. 2015;4:1

22. Sikri VK. Color: implications in dentistry. J Conserv Dent. 2010;13:249-55.

23. Armijo-Olivo S, Stiles CR, Hagen NA, et al. Assessment of study quality for systematic reviews: a comparison of the Cochrane Collaboration Risk of Bias Tool and the Effective Public Health Practice Project Quality Assessment Tool: methodological research. J Eval Clin Pract. 2012;18:12-8.

24. Higgins JP, Green S, editors. Cochrane handbook for systematic reviews of interventions. London: The Cochrane Collaboration; 2011.
25. Balshem H, Helfand M, Schunemann HJ, et al. GRADE guidelines: 3. Rating the quality of evidence. J Clin Epidemiol. 2011;64:401-6.

26. Asahina A, Watanabe T, Kishi A, et al. Evaluation of the treatment of port-wine stains with the 595-nm long pulsed dye laser: a large prospective study in adult Japanese patients. J Am Acad Dermaol. 2006;54:487-93.

27. Ackermann G, Hartmann M, Scherer K, et al. Correlations between light penetration into skin and the therapeutic outcome following laser therapy of port-wine stains. Lasers Med Sci. 2002;17:70-8.

28. Ahčan U, Zorman P, Recek D, et al. Port wine stain treatment with a dual-wavelength $\mathrm{Nd}$ : Yag laser and cryogen spray cooling: a pilot study. Lasers Surg Med. 2004;34:164-7.

29. Al-Dhalimi MA, Al-Janabi MH. Split lesion randomized comparative study between long pulsed Nd:YAG laser 532 and 1,064 $\mathrm{nm}$ in treatment of facial port-wine stain. Lasers Surg Med. 2016;48:852-8.

30. Al-janabi MH, Ismaeel Ali NT, Mohamed Al-Sabti KD, et al. A new imaging technique for assessment of the effectiveness of long pulse Nd: YAG $532 \mathrm{~nm}$ laser in treatment of facial port wine stain. J Cosmet Laser Ther. 2017;9:418-21.

31. Anolik R, Newlove T, Weiss ET, et al. Investigation into optimal treatment intervals of facial port-wine stains using the pulsed dye laser. J Am Acad Dermatol. 2012;67:985-90.

32. Baghad B, Chiheb S, Benchikhi H. Port wine stains and Pulsed Dye Laser: study of prognostic factors in 74 Moroccan patients. Pan Afr Med J. 2016;25:218.

33. Chang CJ, Kelly KM, Van Gemert MJ, Nelson JS. Comparing the effectiveness of 585-nm vs. 595-nm wavelength pulsed dye laser treatment of port wine stains in conjunction with cryogen spray cooling. Lasers Surg Med. 2002;31:352-8.

34. Chapas AM, Eickhorst K, Geronemus RG. Efficacy of early treatment of facial port wine stains in newborns: a review of 49 cases. Lasers Surg Med. 2007;39:563-8.

35. Del Pozo J, Fonseca E. Port-wine stain nodules in the adult: report of 20 cases treated by $\mathrm{CO} 2$ laser vaporization. Dermatol Surg. 2001;27:699-702.

36. Dong X, Yu Q, Ding J, Lin J. Treatment of facial port-wine stains with a new intense pulsed light source in Chinese patients. J Cosmet Laser Ther. 2010;12:183-7.

37. Rehman F-U, Rashid T, Hussain I, Haroon TS. Efficacy and safety of quasi-continuous, frequency-doubled Nd: YAG (532nm) laser therapy of port-wine stains. J Pak Assoc Dermatolog. 2007;17:159-65.

38. Fallahi M, Hallaji Z, Tavakolpour S, et al. Evaluating the efficacy and safety of topical sirolimus $0.2 \%$ cream as adjuvant therapy with pulsed dye laser for the treatment of port wine stain: a randomized, double-blind, placebo-controlled trial. J Cosmet Dermatol. 2020. https://doi.org/10.1111/jocd.13867.

39. Geronemus RG, Quintana AT, Lou WW, Kauvar AN. High-fluence modified pulsed dye laser photocoagulation with dynamic cooling of port-wine stains in infancy. AMA Arch Derm. 2000;136:942-3.

40. Grabb WC, MacCollum M, Tan NG. Results from tattooing portwine hemangiomas: a long-term follow-up. Plast Reconstr Surg. 1977;59:667-9.

41. Greve B, Hammes S, Raulin C. The effect of cold air cooling on $585 \mathrm{~nm}$ pulsed dye laser treatment of port-wine stains. Dermatol Surg. 2001;27:633-6.

42. Huerta-Rivera G, López-Zaldo JB, Arreola-Jáuregui IE, et al. Port wine stain, treatment in a series of cases with pulsed dye laser, the gold standard. Dermatol Rev Mex. 2019;63:8-13.

43. Huikeshoven M, Koster PH, de Borgie CA, et al. Redarkening of port-wine stains 10 years after pulsed-dye-laser treatment. N Engl J Med. 2007;356:1235-40. 
44. Jeon H, Bernstein LJ, Belkin DA, et al. Pulsed dye laser treatment of port-wine stains in infancy without the need for general anesthesia. JAMA Dermatol. 2019;155:435-41.

45. Kelly KM, Nanda VS, Nelson JS. Treatment of port-wine stain birthmarks using the $1.5-\mathrm{msec}$ pulsed dye laser at high fluences in conjunction with cryogen spray cooling. Dermatol Surg. 2002;28:309-13.

46. Koster PH, van der Horst CM, Bossuyt PM, van Gemert MJ. Prediction of portwine stain clearance and required number of flashlamp pumped pulsed dye laser treatments. Lasers Surg Med. 2001;29:151-5.

47. Kono T, Frederick Groff W, Chan HH, et al. Long-pulsed neodymium: yttrium-aluminum-garnet laser treatment for hypertrophic port-wine stains on the lips. J Cosmet Laser Ther. 2009;11:11-3.

48. Kwiek B, Rożalski M, Kowalewski C, Ambroziak M. Retrospective single center study of the efficacy of large spot $532 \mathrm{~nm}$ laser for the treatment of facial capillary malformations in 44 patients with the use of three-dimensional image analysis. Lasers Surg Med. 2017;49:743-9.

49. Léauté-Labréze C, Boralevi F, Pedespan JM, et al. Pulsed dye laser for Sturge-Weber syndrome. Arch Dis Child. 2002;87:434-5.

50. Li D, Chen B, Zhang H, et al. Retrospective study of the treatment of port-wine stains with 595-nm pulsed dye laser in 261 Chinese patients. Lasers Med Sci. 2020;35:1811-9.

51. Li-Qiang G, Hua W, Si-Li N, Chun-Hua T. A clinical study of HMME-PDT therapy in Chinese pediatric patients with port-wine stain. Photodiagn Photodyn Ther. 2018;23:102-5.

52. Ma G, Han Y, Ying H, et al. Comparison of two generation photosensitizers of psd-007 and hematoporphyrin monomethyl ether photodynamic therapy for treatment of port-wine stain: a retrospective study. Photobiomodul Photomed Laser Surg. 2019;37:376-80.

53. Mohamed EE, Mohamed Tawfik K, Hassan AW. Successful treatment of facial vascular skin diseases with a 577-nm pro-yellow laser. J Cosmet Dermatol. 2019;18:1675-9.

54. Özdemir M, Engin B, Mevlitoğlu İ. Treatment of facial port-wine stains with intense pulsed light: a prospective study. J Cosmet Dermatol. 2008;7:127-31.

55. Passeron T, Maza A, Fontas E, et al. Treatment of port wine stains with pulsed dye laser and topical timolol: a multicenter randomized controlled trial. Br J Dermatol. 2014;170:1350-3.

56. Pençe B, Aybey B, Ergenekon G. Outcomes of $532 \mathrm{~nm}$ frequencydoubled Nd:YAG laser use in the treatment of port-wine stains. Dermatol Surg. 2005;31:509-17.

57. Periyasamy MK, Sekar CS, Rai R. Effectiveness of dual sequential wavelength laser in the treatment of portwine stains: a retrospective study. Indian Dermatol Online J. 2019;10:418.

58. Cao KP, Quang MN, Nguyen QD, et al. Anatomical evaluation for successful dye laser treatment of port wine stain in vietnamese patients. Open Access Maced J Med Sci. 2019;7:208-10.

59. Ren J, Li P, Zhao H, et al. Assessment of tissue perfusion changes in port wine stains after vascular targeted photodynamic therapy: a short-term follow-up study. Lasers Med Sci. 2014;29:781-8.

60. Ren J, Qian H, Xiang L, et al. The assessment of pulsed dye laser treatment of port-wine stains with reflectance confocal microscopy. J Cosmet Laser Ther. 2013;16:21-5.

61. Sadeghinia A, Moghaddas S, Tavakolpour S, et al. Treatment of port wine stains with 595 -nm pulsed dye laser in 27 pediatric patients: a prospective study in the Iranian population. J Cosmet Laser Ther. 2019;21:373-7.

62. Sharma VK, Khandpur S. Efficacy of pulsed dye laser in facial port-wine stains in Indian patients. Dermatol Surg. 2007;33:560-6.

63. Sommer S, Sheehan-Dare RA. Pulsed dye laser treatment of port-wine stains in pigmented skin. J Am Acad Dermatol. 2000;42:667-71.
64. Thomson HG, Wright AM. Surgical tattooing of the port-wine stain: operative technique, results, and critique. Plast Reconstr Surg. 1971;48:113-20.

65. van der Velden EM, de Jong BD, van der Walle HB, et al. Cosmetic tattooing as a treatment of port-wine stains. Int J Dermatol. 1993;32:372-5.

66. Wang B, Wu Y, Zhu X, et al. Treatment of neck port-wine stain with intense pulsed light in Chinese population. J Cosmet Laser Ther. 2013;15:85-90.

67. Wang T, Chen D, Yang J, et al. Safety and efficacy of dual-wavelength laser $(1064+595 \mathrm{~nm})$ for treatment of non-treated portwine stains. J Eur Acad Dermatol Venereol. 2018;32:260-4.

68. Wen L, Zhang Y, Zhang L, et al. Application of different noninvasive diagnostic techniques used in HMME-PDT in the treatment of port wine stains. Photodiagnosis Photodyn Ther. 2019;25:369-75.

69. Wimmershoff MB, Wenig M, Hohenleutner U, Landthaler M. Treatment of portwine stains with the flashlamp pumped pulsed dye laser: five years of clinical experience. Hautarzt. 2001;52:1011-5.

70. Xiao Q, Li Q, Yuan KH, Cheng B. Photodynamic therapy of port-wine stains: long-term efficacy and complication in Chinese patients. J Dermatol. 2011;38:1146-52.

71. Yang MU, Yaroslavsky AN, Farinelli WA, et al. Long-pulsed neodymium: yttrium-aluminum-garnet laser treatment for port-wine stains. J Am Acad Dermatol. 2005;52:480-90.

72. Yuan KH, Li Q, Yu WL, et al. Comparison of photodynamic therapy and pulsed dye laser in patients with port wine stain birthmarks: a retrospective analysis. Photodiagnosis Photodyn Ther. 2008;5:50-7.

73. Yu W, Ma G, Qiu Y, et al. Why do port-wine stains (PWS) on the lateral face respond better to pulsed dye laser (PDL) than those located on the central face? J Am Acad Dermatol. 2016;74:527-35.

74. Yu W, Zhu J, Gu Y, et al. Port-wine stains on the neck respond better to a pulsed dye laser than lesions on the face: an intrapatient comparison study with histopathology. J Am Acad Dermatol. 2019;80:779-81.

75. Zhang B, Zhang TH, Huang Z, et al. Comparison of pulsed dye laser (PDL) and photodynamic therapy (PDT) for treatment of facial port-wine stain (PWS) birthmarks in pediatric patients. Photodiagn Photodyn Ther. 2014;11:491-7.

76. Zhong SX, Liu YY, Yao L, et al. Clinical analysis of port-wine stain in 130 Chinese patients treated by long-pulsed 1064-nm Nd:YAG laser. J Cosmet Laser Ther. 2014;16:279-83.

77. Lee JW, Chung HY. Capillary malformations (portwine stains) of the head and neck: natural history, investigations, laser, and surgical management. Otolaryngol Clin N Am. 2018;51:197-211.

78. Dasgupta R. Surgical management of vascular anomalies. Curr Otorhinolaryngol Rep. 2014;2:285-91.

79. van Raath MI, van Amersfoort JE, Hermann M, et al. Site-specific pharmaco-laser therapy: a novel treatment modality for refractory port wine stains. J Clin Transl Res. 2019;5:1-24.

80. Lipner SR. Topical adjuncts to pulsed dye laser for treatment of port wine stains: review of the literature. Dermatol Surg. 2018;44:796-802.

81. Faurschou A, Olesen AB, Leonardi-Bee J, Haedersdal M. Lasers or light sources for treating port-wine stains. Cochrane Database Syst Rev. 2011;11:CD007152.

82. Ashinoff R, Geronemus RG. Flashlamp-pumped pulsed dye laser for port-wine stains in infancy: earlier versus later treatment. J Am Acad Dermatol. 1991;24:467-72.

83. Liu X, Fan Y, Huang J, et al. Can we predict the outcome of 595-nm wavelength pulsed dye laser therapy on capillary vascular malformations from the first beginning: a pilot study of efficacy co-related factors in 686 Chinese patients. Lasers Med Sci. 2015;30:1041-6. 
84. van der Horst CM, Koster PH, de Borgie CA, et al. Effect of the timing of treatment of port-wine stains with the flash-lamppumped pulsed-dye laser. N Engl J Med. 1998;338:1028-33.

85. Bae YS, Ng E, Geronemus RG. Successful treatment of two pediatric port wine stains in darker skin types using $595 \mathrm{~nm}$ laser. Lasers Surg Med. 2016;48:339-42.

86. Chen JK, Ghasri P, Aguilar G, et al. An overview of clinical and experimental treatment modalities for port wine stains. J Am Acad Dermatol. 2012;67:289-304.

87. Hohenleutner U, Hilbert M, Wlotzke U, Landthaler M. Epidermal damage and limited coagulation depth with the flashlamppumped pulsed dye laser: a histochemical study. J Invest Dermatol. 1995;104:798-802.
88. Tong AK, Tan OT, Boll J, et al. Ultrastructure: effects of melanin pigment on target specificity using a pulsed dye laser $(577 \mathrm{~nm})$. J Invest Dermatol. 1987;88:747-52.

89. van Raath MI, Bambach CA, Dijksman LM, et al. Prospective analysis of the port-wine stain patient population in the Netherlands in light of novel treatment modalities. J Cosmet Laser Ther. 2018;20:77-84.

90. Prinsen CAC, Spuls PI, Kottner J, et al. Navigating the landscape of core outcome set development in dermatology. J Am Acad Dermatol. 2019;81:297-305.

\section{Authors and Affiliations}

\section{Gonca Cinkara ${ }^{1} \cdot$ Ginger Beau Langbroek ${ }^{2} \oplus$ - Chantal M. A. M. van der Horst ${ }^{3}$ - Albert Wolkerstorfer ${ }^{4}$. Sophie E. R. Horbach ${ }^{3}$. Dirk T. Ubbink ${ }^{2}$}

1 Faculty of Medicine, University of Amsterdam, Amsterdam, The Netherlands

2 Department of Surgery, Amsterdam University Medical Centers, Location AMC, P.O. Box 22660, 1100 DD Amsterdam, The Netherlands
3 Department of Plastic, Reconstructive and Hand Surgery, Amsterdam University Medical Centers, Location AMC, Amsterdam, The Netherlands

4 Department of Dermatology, Amsterdam University Medical Centers, Location AMC, Amsterdam, The Netherlands 